\title{
ISOMORPHIC FACTORISATIONS. I: COMPLETE GRAPHS
}

BY

\author{
FRANK HARARY, ROBERT W. ROBINSON AND NICHOLAS C. WORMALD
}

\begin{abstract}
An isomorphic factorisation of the complete graph $K_{p}$ is a partition of the lines of $K_{p}$ into $t$ isomorphic spanning subgraphs $G$; we then write $G \mid K_{p}$ and $G \in K_{p} / t$. If the set of graphs $K_{p} / t$ is not empty, then of course $t \mid p(p-1) / 2$. Our principal purpose is to prove the converse. It was found by Laura Guidotti that the converse does hold whenever $(t, p)=1$ or $(t, p-1)=1$, We give a new and shorter proof of her result which involves permuting the points and lines of $K_{p}$. The construction developed in our proof happens to give all the graphs in $K_{6} / 3$ and $K_{7} / 3$. The Divisibility Theorem asserts that there is a factorisation of $K_{p}$ into $t$ isomorphic parts whenever $t$ divides $p(p-1) / 2$. The proof to be given is based on our proof of Guidotti's Theorem, with embellishments to handle the additional difficulties presented by the cases when $t$ is not relatively prime to $p$ or $p-1$.
\end{abstract}

1. Introduction. A factorisation of a graph $H$ is a partition of its lines into disjoint classes. Each class of lines in a factorisation of $H$ corresponds to some spanning subgraph, called a factor. A factorisation in which all of the factors are isomorphic to each other is called an isomorphic factorisation. Let $H / t$ denote the set of graphs which occur as factors in isomorphic factorisations of $H$ into exactly $t$ factors. If $G$ is such a graph, we write $G \mid H$ and we say that $G$ divides $H$. We also say $H$ is divisible by $t$, written $t \mid H$, if $H / t$ is not empty.

If $H$ has $q$ lines, then of course $H / t$ will be empty unless $t \mid q$. In general this necessary condition is not sufficient. Figure 1 shows the subdivision graph $T$ of the star $K_{1,3}$. This has 6 lines and yet $T / 2$ is empty.

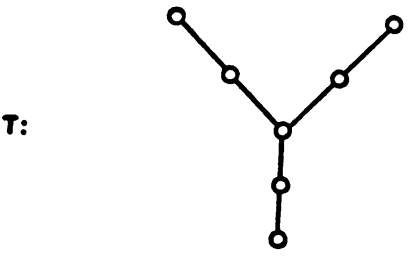

FIGURE 1. A tree not divisible by 2

For all graph theoretic terminology and notation, we follow the book [2]. In

Received by the editors November 22, 1976 and, in revised form, April 4, 1977.

AMS (MOS) subject classifications (1970). Primary 05C99; Secondary 05B30.

Key words and phrases. Factorisations, complete graphs.

- American Mathematical Society 1978 
particular, a graph will always be finite and undirected, with no loops or multiple edges. The complement of $G$ is written $\bar{G}$. The complete graph with $p$ points is $K_{p}$ but when $p$ is given by a complicated expression we will write $K(p)$. Similarly the complete bipartite graph is denoted by $K_{m, n}$ or $K(m, n)$.

In this paper we study isomorphic factorisations of complete graphs. Our main object is to show that the obvious necessary condition $t \mid p(p-1) / 2$ for $K_{p}$ to be divisible by $t$ is also sufficient. This is implied by the following theorem, which is proved in $\$ 4$.

Divisibility THeOREM. The complete graph $K_{p}$ is divisible by $t$ if and only if $t$ divides $p(p-1) / 2$.

We first prove, in $\S 2$, that this is true when $t$ is relatively prime to $p$ or $p-1$. The method of construction employed in this proof is extended, using some clever ideas contributed by the third author, so as to handle arbitrary $t$ and $p$.

The members of $K_{p} / 2$ are well known under the same name of self-complementary graphs. These have been investigated extensively, for example in [10], [11] and [15]. As a beginning to the study of $K_{p} / 3$, in $\$ 3$ we exhibit explicitly all the graphs in $K_{6} / 3$ and $K_{7} / 3$. We conclude the paper with a survey of other results on isomorphic factorisations of complete graphs.

2. A sufficient condition for the existence of an isomorphic factorisation. When does there exist a graph $G$, say with $q$ lines, in $K_{p} / t$ ? Obviously the existence of $G$ implies $t q=p(p-1) / 2$ so that $p(p-1) / 2 t$ is an integer.

The first result in the converse direction is due to Guidotti [2] and shows that under certain restrictive number theoretic conditions, the Divisibility Theorem does hold.

THEOREM 1. If $t \mid p(p-1) / 2$ and $(t, p)=1$ or $(t, p-1)=1$, then $K_{p}$ is divisible by $t$.

This result provides a striking example of independent discovery in graph theory. For we proposed the question to J. Schönheim and he found and proved the theorem. Then we were sent the article by Guidotti [2] to digest for Mathematical Reviews and we found that she had already published it! We now provide a third proof of the same proposition. Our approach is a natural extension of the method of construction of self-complementary graphs discovered by Sachs [15] and Ringel [11]-another example of independent discovery in graph theory. Our proof gives a method of constructing isomorphic factorisations which will be the basis for the proof of the Divisibility Theorem, and will also produce all of the graphs in $K_{6} / 3$ and $K_{7} / 3$.

PROOF OF THEOREM 1. We consider three cases separately.

Case 1. $(t, p-1)=1$ and $t$ is odd. It is convenient to write $V=$ 
$\{1,2, \ldots, p\}$ for the point set of $K_{p}$. Since $t \mid p(p-1) / 2$ and $(t, p-1)=1$, it follows that $t \mid p$. The trick is to consider only those permutations of $V$ in which every cycle length is a multiple of $t$. Let $\phi$ be such a permutation. We now need the corresponding permutation $\phi^{\prime}$ which acts on the lines of $K_{p}$. This is exactly the permutation in the pair group of the symmetric group $S_{p}$ defined by $\phi^{\prime}\{i, j\}=\{\phi i, \phi j\}$; see [4, p. 83], where this pair group is seen to be useful in the enumeration of graphs.

We now show that the induced permutation $\phi^{\prime}$ will likewise have every cycle length a multiple of $t$. There are two possibilities for each line in $K_{p}$ : it either joins points in two different cycles of $\phi$, say of lengths $a$ and $b$, or it joins two points in the same cycle of $\phi$, say of length $c$. In the first case all of the lines joining pairs in the two cycles in $\phi$ of length $a$ and $b$ are permuted in cycles of $\phi^{\prime}$ of length $[a, b]$. Of course, $t \mid[a, b]$ as $t \mid a$. In the second case all of the lines joining points in the cycle of length $c$ in $\phi$ will be permuted by $\phi^{\prime}$ in cycles of length $c$, unless $c$ is even when there is a single "diagonal" cycle of lines of length $c / 2$. Now $t \mid c$, and if $c$ is even $t \mid(c / 2)$ since $t$ is odd.

We are now ready to construct a graph $G$ which divides $K_{p}$ into $t$ parts. This is done by exploiting the induced permutation $\phi^{\prime}$. Let $\phi^{\prime}$ be written as a product of cycles, $\phi^{\prime}=\gamma_{1} \gamma_{2} \cdots \gamma_{r}$. From each cycle $\gamma_{i}$ choose a line $e_{i} \in \gamma_{i}$. Let $E=E(G)=\left\{\left(\phi^{\prime}\right)^{n t}\left(e_{i}\right) \mid n \geqslant 0,1 \leqslant i \leqslant r\right\}$. Then $\left\{E, \phi^{\prime} E, \ldots,\left(\phi^{\prime}\right)^{t-1} E\right\}$ is a partition of the lines of $K_{p}$. This constitutes an isomorphic factorisation of $K_{p}$, as the subgraph $G$ induced by $E$ is isomorphic to the subgraphs of $K_{p}$ induced by each of $\phi^{\prime} E, \ldots$ Isomorphisms between $G$ and these subgraphs are provided by the corresponding powers of $\phi$. Hence $G \in K_{p} / t$.

Case 2. $(t, p-1)=1$ and $t$ is even. In this case $p-1$ is odd, and so $t \mid p(p-1) / 2$ implies that $2 t \mid p$. Take a permutation $\phi$ of $V$ for which the length of every cycle is a multiple of $2 t$. The induced permutation $\phi^{\prime}$ is seen to have the property that the length of every cycle is divisible by $t$. We now apply the same construction as in Case 1 to obtain a graph in $K_{p} / t$.

Case 3. $(t, p)=1$. In this case $p \equiv 1(\bmod t)$ if $t$ is odd and $p \equiv 1(\bmod 2 t)$ if $t$ is even. Take a permutation $\phi$ acting on $V$ with just one fixed point. If $t$ is odd, let all other cycles of $\phi$ have lengths which are multiples of $t$; if $t$ is even let these lengths be multiples of $2 t$. The induced permutation $\phi^{\prime}$ has all cycle lengths divisible by $t$. The same construction works again to provide a graph in $K_{p} / t$.

3. All the graphs which tridivide $K_{6}$ and $K_{7}$. We say that $G$ tridivides $H$ if $G \in H / 3$. When $H=K_{p}$ and $t=3$, the condition that $p(p-1) / 2 t$ is an integer holds if and only if $p \equiv 0$ or $1(\bmod 3)$. The smallest nontrivial cases are for $p=3$ and 4 . It is obvious that

$$
\begin{aligned}
& K_{3} / 3=\left\{K_{2} \cup K_{1}\right\} \text { and } \\
& K_{4} / 3=\left\{2 K_{2}, K_{1,2} \cup K_{1}\right\} .
\end{aligned}
$$


It is immediately seen that the method of Theorem 1 yields the graph in $K_{3} / 3$ and both graphs in $K_{4} / 3$. The next nontrivial cases are for $p=6$ and 7 . We will find that Theorem 1 gives all the graphs in $K_{6} / 3$ and $K_{7} / 3$. These graphs had already been listed by A. Bialostocki and J. Schönheim using heuristic methods.

Before enumerating all these graphs, we give the details of the construction of one of the graphs in $K_{6} / 3$, namely the path $P_{6}$, in order to illustrate the method of Theorem 1.

Following the proof, we write $V=\{1,2,3,4,5,6\}$ for the point set of $K_{6}$. We now choose a permutation $\phi$ on $V$ in which every cycle length is a multiple of 3 , namely

$$
\phi=\left(\begin{array}{lll}
1 & 2 & 3
\end{array}\right)\left(\begin{array}{lll}
4 & 5 & 6
\end{array}\right) \text {. }
$$

It is convenient to write the 15 lines in $K_{6}$ in the concise form $\{12,13,14, \ldots, 56\}$ in which 12 stands for $\{1,2\}$, etc. Using this notation the induced permutation on the lines of $K_{6}$ takes the form

$$
\phi^{\prime}=\left(\begin{array}{l}
12 \\
23
\end{array} 13\right)(455646)(152634)(142536)(162435) \text {. }
$$

In accordance with the notation in the proof of Theorem 1, we refer to the five cycles in $\phi^{\prime}$ as $\gamma_{1}, \ldots, \gamma_{5}$. The next step in the proof is to choose one line from each of the five cycles, and we select $13,45,15,36$ and 24 . As each cycle in $\phi^{\prime}$ has length $3=t$, this set is $E(G)$. Thus $G$ is a path $P_{6}$.

To display this construction in Figure 2 we use dotted directed lines to represent the permutation $\phi$ acting on the points, and solid lines for the chosen edge set of $G$.

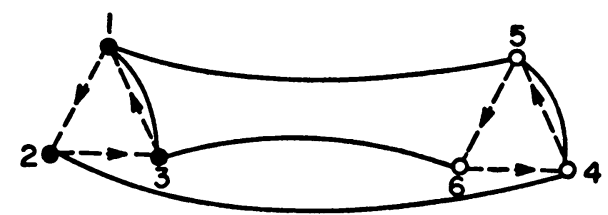

FIGURE 2. Construction showing that $P_{6}$ is in $K_{6} / 3$

Applying $\phi^{\prime}$ to the lines of $G$ twice, we obtain

$$
\begin{aligned}
\phi^{\prime} E & =\{12,56,26,14,35\} \text { and } \\
\left(\phi^{\prime}\right)^{2} E & =\{23,46,34,25,16\} .
\end{aligned}
$$

Of course both of these edge sets also give copies of $P_{6}$. As $E, \phi^{\prime} E$, and $\left(\phi^{\prime}\right)^{2} E$ partition the lines of $K_{6}$, we see that $P_{6} \mid K_{6}$.

We now show how to construct all the graphs in $K_{6} / 3$ which can be obtained by this method. There are just two possible cycle structures for $\phi$, namely a 6-cycle or the product of two disjoint 3-cycles. However any graph in $K_{6} / 3$ obtained from a 6 -cycle can also be obtained from the square of the 
6-cycle, which is a product of 3-cycles. Consider all possible choices of one line from each of the five cycles in $\phi^{\prime}$ used in the above construction of $P_{6}$. Up to isomorphism these exhaust all of the graphs in $K_{6} / 3$ which can be obtained by the method of Theorem 1 .

It is not necessary to consider all such possible choices, as one line from

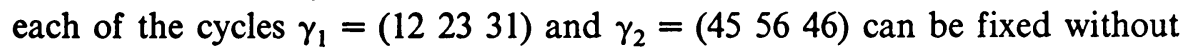
loss of generality. That leaves only 27 choices from the other three cycles. However it is easy to verify that these lead to just nine different graphs in $K_{6} / 3$. These are shown in Figure 3, in which the points are solid or open, as determined by some construction in which 1, 2 and 3 are solid and 4, 5 and 6 are open.
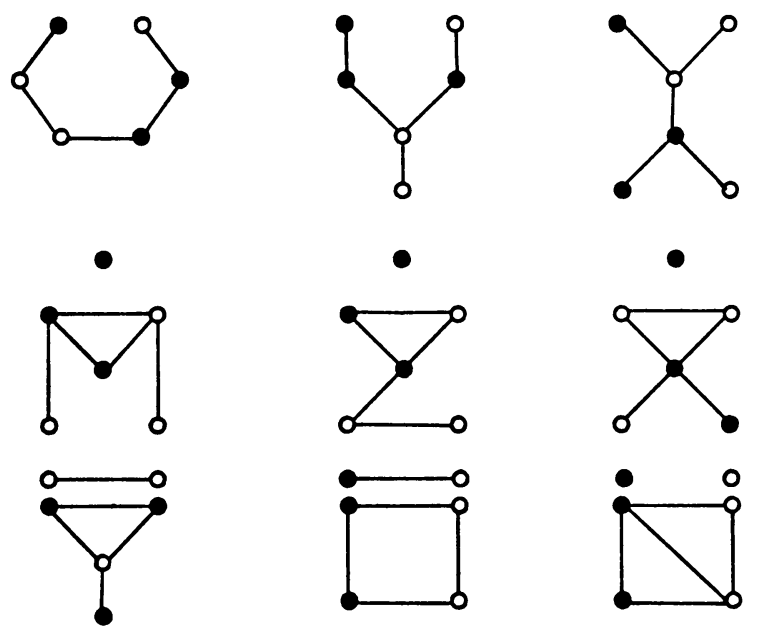

FIGURE 3. The graphs in $K_{6} / 3$

All the graphs with 6 points and 5 lines are shown in [3, p. 219]. In addition to the nine graphs in Figure 3, there are just six others. It is routine to check that none of these is in $K_{6} / 3$.

THEOREM 2. The set $K_{6} / 3$ consists of the nine graphs of Figure 3 .

For the construction of graphs in $K_{7} / 3$, we can use without loss of generality the permutation $\alpha=\left(\begin{array}{ll}1 & 23\end{array}\right)\left(\begin{array}{ll}4 & 5\end{array}\right)(7)$, which is just $\phi$ extended by a fixed point, 7. Then $\alpha^{\prime}$ is just the product of $\phi^{\prime}$ with (17 27 37)(47 57 67). Instead of 27 choices of edge sets we now have $3^{5}=243$ possible choices to consider. These reduce to the 41 graphs shown in Figure 4, in which the fixed point 7 is shown as open. It happens that each of the 41 graphs can be obtained from one of those shown in Figure 3 by joining 7 to one open and one solid point. 
It is known that there are exactly 65 graphs on 7 points with 7 lines. It is straightforward but tedious to verify that none of the other 24 is in $K_{7} / 3$.

THEOREM 3. The set $K_{7} / 3$ consists of the 41 graphs of Figure 4.
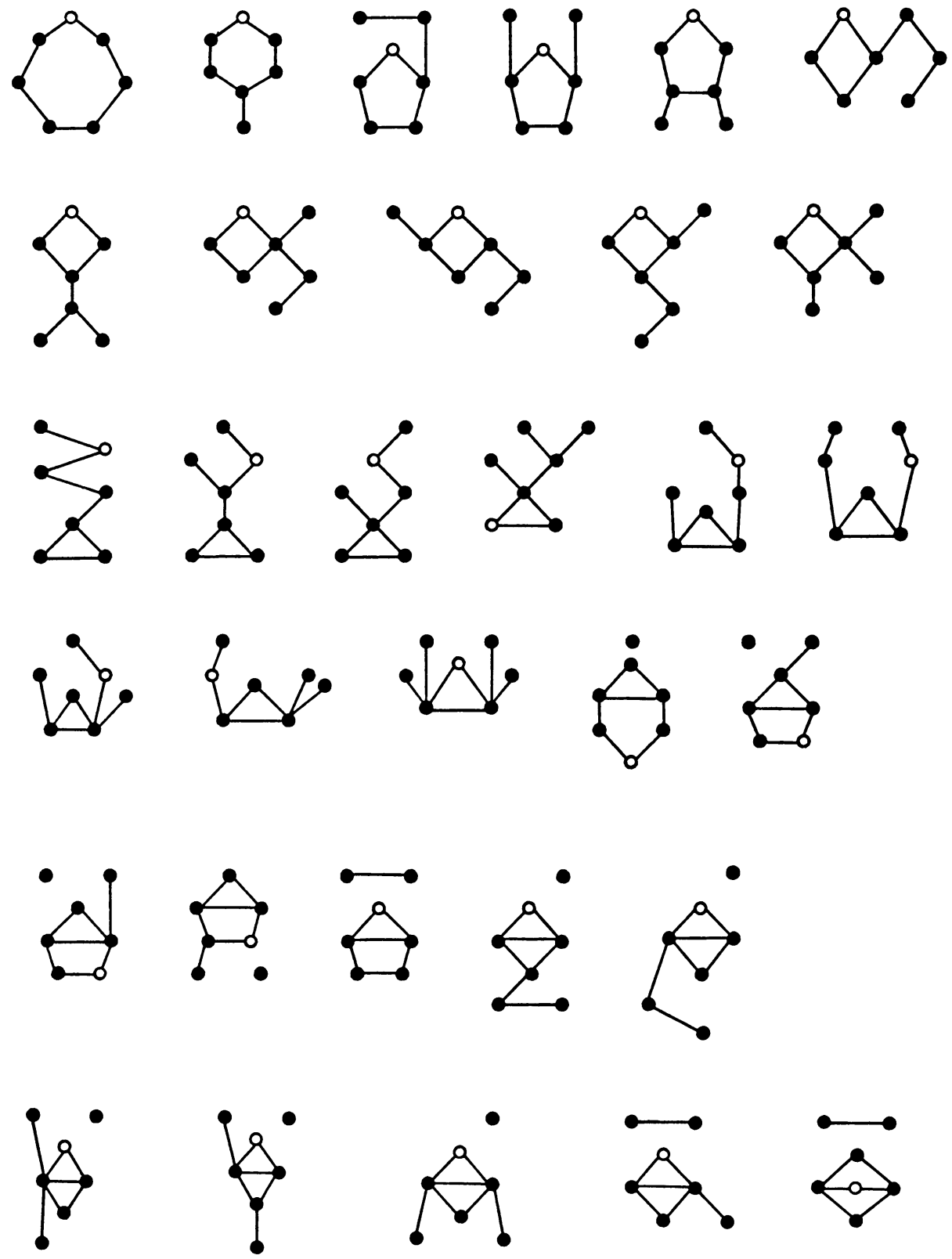

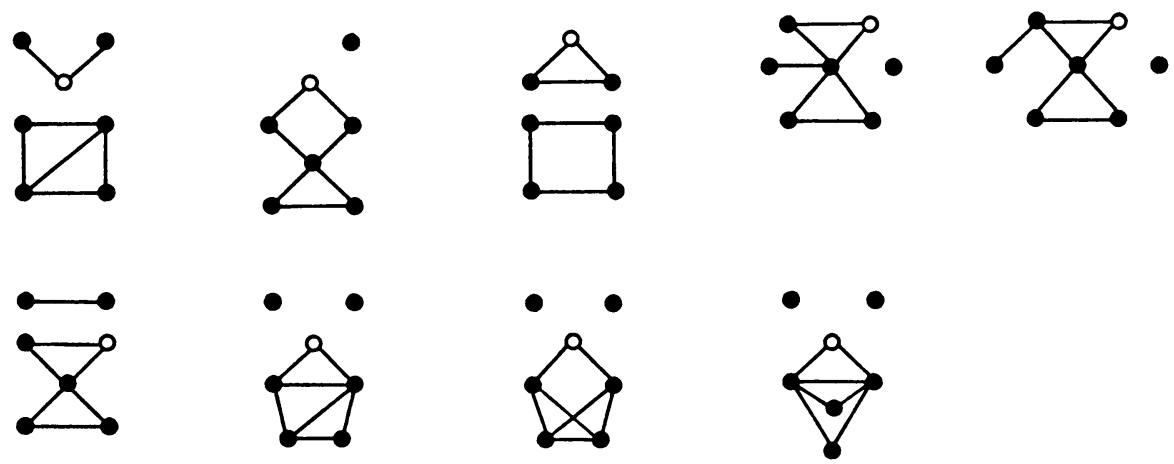

FIGURE 4. The graphs in $K_{7} / 3$

\section{The main theorem.}

Theorem 4 (Divisibility TheOREM). The complete graph $K_{p}$ is divisible by $t$ if and only if $t$ divides $p(p-1) / 2$.

In Theorem 1 we proved a special case of the Divisibility Theorem. Our present purpose is to prove this theorem in full generality.

As the proof is rather complicated, we will develop it in the form of three lemmas. When $t$ is odd the first two lemmas serve to complete the proof. When $t$ is even the third lemma is also required. Lemma 1 uses the method of Theorem 1. After it has been demonstrated, we will only need to prove Theorem 4 when $p<t$ if $t$ is odd and $p<2 t$ if $t$ is even. Lemma 2 provides a factorisation of $K_{p}$ into factors of the form $q K_{2}$ plus isolates when $p<t$, where $t q=p(p-1) / 2$.

LEMMA 1. Let $t \mid K_{r}$ and $p>r$.

(i) If $t$ is odd and $p \equiv r(\bmod t)$ then $t \mid K_{p}$.

(ii) If $t$ is even and $p \equiv r(\bmod 2 t)$ then $t \mid K_{p}$.

Proof of (i). Let $n t=p-r$, and let $V_{p}=\{1,2, \ldots, p\}$ be the point set of $K_{p}$. We consider the complete subgraph $K_{n t}$ on the point set $V_{n t}=$ $\{1,2, \ldots, n t\}$. In Theorem 1, an appropriate factorisation of $K_{n t}$ was obtained using any permutation $\phi$ of $V_{n t}$ whose cycles have lengths divisible by $t$. For the sake of simplicity we now take $\phi$ as a product of $n$ disjoint cycles, each of length $t$. As in Case 1 of Theorem 1, $\phi^{\prime}$ denotes the permutation induced by $\phi$ on the lines of $K_{n t}$. Since $t$ is odd, every cycle of $\phi^{\prime}$ has length $t$. Hence, by choosing exactly one line from each cycle of $\phi^{\prime}$ to form the initial set $E^{\prime}$, we obtain a partition $\left\{E^{\prime}, \phi^{\prime} E^{\prime}, \ldots,\left(\phi^{\prime}\right)^{t-1} E^{\prime}\right\}$ of the lines of $K_{n t}$. If $G^{\prime}$ is the subgraph induced by $E^{\prime}$, then as before we have $G^{\prime} \in K_{n t} / t$.

Consider the complete graph $K_{r}$ on the point set $\{n t+1, n t+2, \ldots, p\}$. 
By hypothesis $K_{r}$ is divisible by $t$, so let $\left\{F_{0}, F_{1}, \ldots, F_{t-1}\right\}$ be a factorisation of $K_{r}$ with each factor isomorphic to some graph $H$.

We are now ready to construct an isomorphic factorisation of $K_{p}$. We start by choosing a point $v_{i}$ in each of the $n$ cycles $\gamma_{i}$ of $\phi$. For $0 \leqslant k \leqslant t-1$, consider the set $D_{k}$ of $n r$ lines of $K_{p}$ which join the points of $K_{r}$ with the $n$ points $\phi^{k} v_{i}$. We now form the $t$ sets $E_{k}=D_{k} \cup\left(\phi^{\prime}\right)^{k} E^{\prime} \cup F_{k}$. After pausing to illustrate this construction, we will show that $\left\{E_{0}, E_{1}, \ldots, E_{t-1}\right\}$ is an isomorphic factorisation of $K_{p}$, as required.

We display in Figure 5 a sample construction of $E_{0}$ for $p=21$ and $t=15$, so that $n=1$ and $r=6$. The seven lines shown within $K_{15}$ constitute $E^{\prime}$, the six lines joining point $v_{1}$ of $K_{15}$ with $K_{6}$ form $D_{0}$, and the single line in $K_{6}$ is $F_{0}$. Obviously the only graph in $K_{6} / 15$ is $K_{2}$ plus isolates. It does not matter which line of $K_{6}$ is chosen for $F_{0}$ because of the symmetry of the construction.

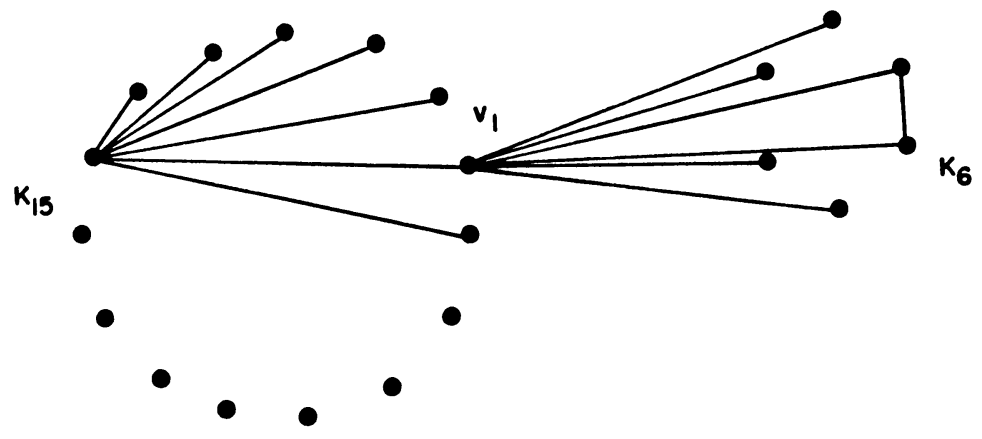

Figure 5. A graph in $K_{21} / 15$

We now return to the proof, and show that the $E_{k}$ partition the lines of $K_{p}$. As the cycles in $\phi$ are all of length $t$, it is clear that the sets $D_{k}$ partition the set of $n t r$ lines joining $K_{n t}$ to $K_{r}$. Thus, since the line sets $\left(\phi^{\prime}\right)^{k} E^{\prime}$ give a factorisation of $K_{n t}$, and the $F_{k}$ are a factorisation of $K_{r}$, it follows that the $E_{k}$ form a factorisation of $K_{p}$.

For $0<k<t-1$, let $G_{k}$ be the spanning subgraph of $K_{p}$ with line set $E_{k}$, and let $J_{k}$ be the factor of $K_{n t}$ induced by $\left(\phi^{\prime}\right)^{k} E^{\prime}$. All that remains to be shown is that $G_{0}$ and $G_{k}$ are isomorphic for $1<k<t-1$. Recall that $H$ is the graph isomorphic to the subgraph of $K_{r}$ induced by each $F_{k}$. But now $G_{0}$ can be regarded as the union of $J_{0}$ and $H$, together with the $n r$ lines joining the points $\left\{v_{1}, v_{2}, \ldots, v_{n}\right\}$ with those of $H$. Similarly, $G_{k}$ is the union of $J_{k}$ and $H$, together with the $n r$ lines joining $\left\{\phi^{k}\left(v_{1}\right), \phi^{k}\left(v_{2}\right), \ldots, \phi^{k}\left(v_{n}\right)\right\}$ with the points of $H$. Since $\phi^{k}$ is an isomorphism from $J_{0}$ to $J_{k}$ which maps $v_{i}$ onto $\phi^{k}\left(v_{i}\right)$ for $i=1$ to $n$, we see that $G_{0}$ and $G_{k}$ are isomorphic. Thus $G_{0} \in K_{p} / t$, and (i) is established.

Proof of (ii). This is done in exactly the same way as in (i), except that 
since $t$ is now even we take for $\phi$ a permutation in which each cycle has length $2 t$. Then as in Case 2 of Theorem 1, the induced line permutation $\phi^{\prime}$ has all its cycle lengths divisible by $t$. The rest of the proof then follows as in (i).

Traditionally, a graph $G$ with $p$ points and $q$ lines is labelled when the numbers $1,2, \ldots, p$ are assigned to its points. Similarly $G$ is line-labelled when $1,2, \ldots, q$ are assigned to its lines. The next Lemma concerns labelling the lines of a complete graph. It will be used directly in constructing divisors of $K_{p}$ when $p<t$. It was previously discovered by .F. Hwang (verbal communication) and by Simmons and Davis [16], in the context of combinatorial designs.

LEMMA 2. The complete graph $K_{m}$ can be line-labelled so that any $n$ consecutive lines are independent, for $n<(m-1) / 2$.

For example, if $m=7$, the largest value for $n$ under consideration is 2 . We display in Figure 6 the beginning of a method of labelling the lines of $K_{7}$ so that no two consecutive lines are adjacent (i.e., have a common point). Note that this consecutivity property entails line-labelling modulo $q$.

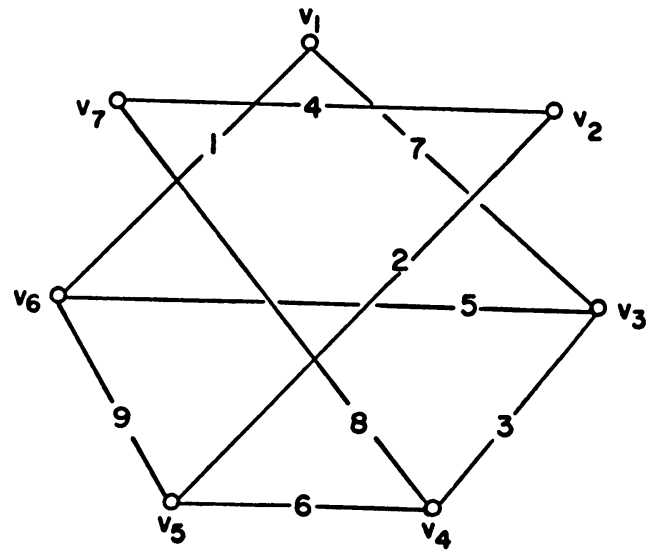

FIGURE 6. Labelling the first 9 lines of $K_{7}$

Proof of Lemma 2. Consider the complete graph $K_{m}$ with point set $V=\left\{v_{1}, \ldots, v_{m}\right\}$. The exact construction to be used will depend on the parity of $m$.

Case 1. $m$ is odd. Let $\phi$ be a cyclic permutation $\left(v_{1} v_{2} \cdots v_{m}\right)$ of $V$. We label the lines of $K_{m}$ as follows. For $1 \leqslant i \leqslant(m-1) / 2$ and $0 \leqslant j \leqslant m-1$, label the line $\left\{\phi^{j} v_{i}, \phi^{j} v_{m-i}\right\}$ with the number $i+j(m-1) / 2$. Note that the lines in the set $L_{0}=\left\{\left\{v_{1}, v_{m-1}\right\},\left\{v_{2}, v_{m-2}\right\}, \ldots,\left\{v_{(m-1) / 2}, v_{(m+1) / 2}\right\}\right\}$ are labelled $1,2, \ldots,(m-1) / 2$. Since these lines are distinct representatives of the line cycles of the permutation $\phi^{\prime}$ induced by $\phi$, and since each of these 
line cycles has length $m$, this system of labelling associates each line of $K_{m}$ with a unique integer in the required range. It is this system of labelling applied to $K_{7}$ which is shown in Figure 6. Thus each $L_{j}=\left(\phi^{\prime}\right)^{j} L_{0}$ is a set of $(m-1) / 2$ consecutive lines, no two of which are adjacent. Furthermore, if we take any set $L$ consisting of the last $k$ lines of $L_{j}$ and the first $(m-3) / 2$ $-k$ lines of $L_{j+1}$, it is easy to see from the specified system of labelling that no pair of distinct lines of $L$ are adjacent. Thus each set of $(m-3) / 2$ consecutive lines of $K_{m}$ contains no pair of adjacent lines. The number $n$ of consecutive lines which are to be disjoint is specified in the lemma to be less than $(m-1) / 2$, i.e., at most $(m-3) / 2$ since $m$ is odd in Case 1 . Hence Case 1 has been proved.

Case 2. $m$ is even. This time we label the lines of $K_{m}$ using a different system. As in Case 1 let $\phi$ be the cyclic permutation $\left(v_{1} v_{2} \cdots v_{m}\right)$ of $V$. For $1 \leqslant i \leqslant m / 2$ and $0 \leqslant j<m / 2$, assign the integer $i+(m-1) j$ to the line $\left\{\phi^{j} v_{i}, \phi^{j} v_{m+1-i}\right\}$. Then, for $1 \leqslant i<m / 2$ and $0 \leqslant j<m / 2$, assign the integer $m / 2+i+(m-1) j$ to the line $\left\{\phi^{j} v_{i+1}, \phi^{j} v_{m+1-i}\right\}$. Note that the lines in the set

$$
\begin{aligned}
L_{0}=\left\{\left\{v_{1}, v_{m}\right\},\left\{v_{2}, v_{m-1}\right\}\right. & \ldots,\left\{v_{m / 2} v_{m / 2+1}\right\}, \\
\left\{v_{2}, v_{m}\right\} & \left.,\left\{v_{3}, v_{m-1}\right\}, \ldots,\left\{v_{m / 2}, v_{m / 2+2}\right\}\right\}
\end{aligned}
$$

are labelled $1,2, \ldots, m-1$. The sets $L_{i}=\left(\phi^{\prime}\right)^{i} L_{0}$ for $0 \leqslant i<m / 2$ are seen to partition the lines of $K_{m}$. For $0 \leqslant i<m / 2$, the labels associated with $L_{i}$ are obtained from those of $L_{0}$ by adding $i(m-1)$ to each. Thus every line of $K_{m}$ has been assigned a unique integer in the range 1 to $m(m-1) / 2$. Figure 7 shows the start of this labelling when applied to $K_{6}$.

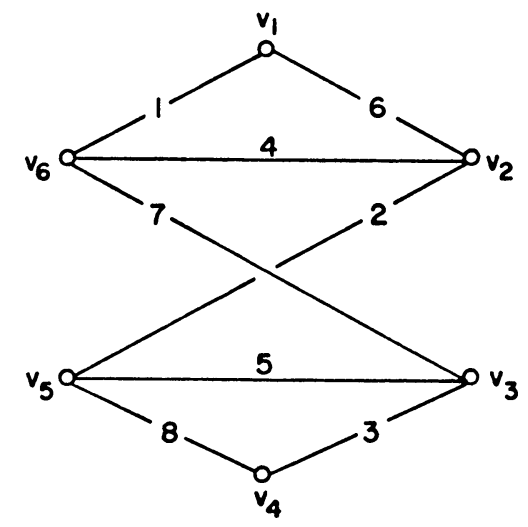

FIGURE 7. Labelling the first 8 lines of $K_{6}$

An inspection, similar to that in Case 1, of this labelling system shows that 
of any $(m-2) / 2$ consecutive lines, no two are adjacent. This completes Case 2 , and therefore Lemma 2 as well.

As a corollary to Lemma 2, we now verify the Divisibility Theorem for odd $t$.

Corollary 1. If $t$ is odd and divides $p(p-1) / 2$, then the complete graph $K_{p}$ is divisible by $t$.

Proof. Assume $t \mid p(p-1) / 2$ and $t$ is odd. Let $r$ be the remainder on dividing $p$ by $t$; thus $0 \leqslant r<t$. Then $t \mid r(r-1) / 2$; write $k t=r(r-1) / 2$. If $k=0$ then $r=0$ or 1 ; so $(t, p)$ or $(t, p-1)$ is 1 and the corollary follows from Theorem 1 . So let $k>0$. Since $t>r$, we also have $k<(r-1) / 2$. Thus by Lemma 2 the lines of $K_{r}$ can be labelled in such a way that of any $k$ consecutive lines, no two are adjacent. Partition the lines of $K_{r}$ into parts $P_{1}, P_{2}, \ldots, P_{t}$ by putting the first $k$ lines in $P_{1}$, the next $k$ in $P_{2}$, and so on, with $\left|P_{i}\right|=k$ for each $i$. Since for each $i$ no two lines within $P_{i}$ are adjacent, this is a factorisation of $K_{r}$ in which every factor is isomorphic to $k K_{2}$ plus isolated points. Thus $K_{r}$ is divisible by $t$, and, so by Lemma $1, K_{p}$ is also divisible by $t$.

Figure 5 shows an illustration of the construction given in the above proof. To find a graph in $K_{21} / 15$, we first use Lemma 2 to find a graph in $K_{6} / 15$, in this case $K_{2} \cup 4 K_{1}$. Then the method of Lemma 1, part (i) is applied to add the remaining lines as shown in the figure.

Note that the proof of Corollary 1 serves to establish the Divisibility Theorem in case $t$ is even and $p \equiv r(\bmod 2 t)$ for $0 \leqslant r<t$. So we suppose that $p \equiv t+r(\bmod 2 t)$ for $0 \leqslant r<t$. By Lemma 1 it suffices to prove the divisibility when $p=t+r$. This is reduced in the following lemma to the problem of finding a certain type of factorisation of $K_{r}$.

LEMMA 3. Let $p, t$ and $r$ be nonnegative integers with $t$ even such that $t>4$, $r=p-t$, and $t>r>1$. Suppose further that there is a graph $G$ (with $r-2$ points) together with a factorisation of $K_{r}$ into $t$ factors consisting of $t / 2$ copies of $G \cup K_{2}$ and $t / 2$ copies of $G \cup 2 K_{1}$. Then $K_{p}$ is divisible by $t$.

Proof. Take the complete graph $K_{p}$ on the point set $V=\{1,2, \ldots, p\}$ and let $K_{t}$ be the complete subgraph with point set $V^{*}=\{1,2, \ldots, t\}$. Let $\phi^{\prime}$ denote the permutation of the lines of $K_{t}$ which is induced by the cyclic permutation $\phi=(1,2 \ldots t)$. Then as we saw in the proof of Theorem 1 , the only cycle of $\phi^{\prime}$ which is not of length $t$ is the "diagonal" cycle of length $t / 2$ which contains the line $\{1,1+t / 2\}$. Write $\phi^{\prime}$ as a product of disjoint cycles $\gamma_{1} \gamma_{2} \cdots \gamma_{t / 2}$ where $\gamma_{t / 2}$ is of length $t / 2$ and all other cycles are of length $t$. Let $S$ be the set of lines $\{\{1,2\},\{1,2+t / 2\},\{2,2+t / 2\},\{1,3\}$, $\{1,4\}, \ldots,\{1, t / 2-1\}\}$. As $t>4$ it is seen that $S$ contains exactly one 
line from each of the cycles of $\phi^{\prime}$. The additional property of $S$ which we will require later is that for two points $i, j$ distinct from 1 (take $i=2, j=2+$ $t / 2),\{i, j\}$ is the representative of $\gamma_{t / 2}$ in $S,\{1, i\}$ and $\{1, j\}$ are in $S$, and $\{i, j\}$ is adjacent to no other lines in $S$.

We are now ready to construct a graph in $K_{p} / t$. Let $K_{r}$ be the complete graph with point set $\{t+1, t+2, \ldots, p\}$, and let $T$ be the set of lines joining the point 1 to the points of $K_{r}$. Next, from the hypothesis of the lemma we may take a factorisation of $K_{r}$ into subgraphs $G_{1}, G_{2}, \ldots, G_{t}$ so that for some fixed graph $G$ we have $G_{1} \cong \cdots \cong G_{t / 2} \cong G \cup 2 K_{1}$ and $G_{1+t / 2} \cong \cdots \cong G_{t} \cong G \cup K_{2}$. Let $F_{1}, F_{2}, \ldots, F_{t}$ be the line sets of $G_{1}, G_{2}, \ldots, G_{t}$. Finally, let $H_{1}$ be the subgraph of $K_{p}$ with line set $E_{1}=S \cup$ $T \cup F_{1}$. After illustrating this construction, we will show that $H_{1} \in K_{p} / t$.

In Figure 8 we exhibit the graph $H_{1}$ obtained when $p=9, t=6$, and $G=K_{1}$. In this instance $S$ must simply consist of the lines of a triangle.

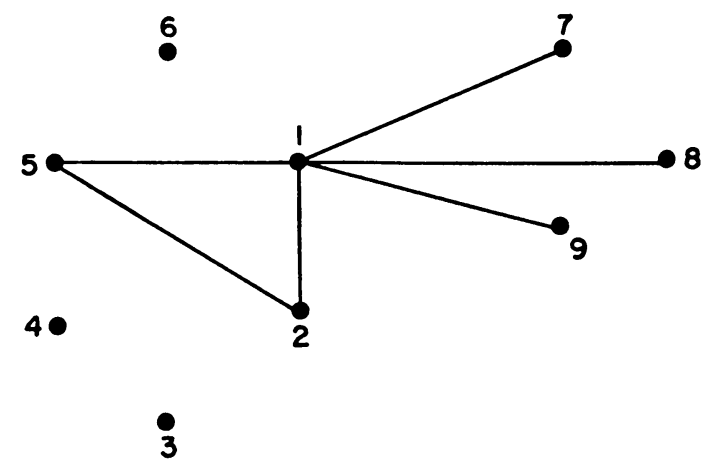

FIGURE 8. A graph in $K_{9} / 6$

For each $1 \leqslant k \leqslant t$ let

$$
S_{k}= \begin{cases}\left(\phi^{\prime}\right)^{k-1} S & \text { if } 1<k<t / 2, \\ \left(\phi^{\prime}\right)^{k-1}(S-\{i, j\}) & \text { if } t / 2<k<t .\end{cases}
$$

Thus half of these are copies of $S$, and the rest have had the "diagonal" line removed in such a way that the $S_{k}$ partition the lines of $K_{t}$.

Next, let $E_{k}$ consist of the lines of $S_{k}$ and $F_{k}$ together with all lines joining a point of $K_{r}$ to the point $k$. It is clear that $E_{1}, \ldots, E_{t}$ partition the lines of $K_{p}$; we will show that this is the required isomorphic factorisation of $K_{p}$. Note the similarity between these $E_{k}$ and those in the proof of Lemma 1, part (i).

Let $H_{k}$ be the subgraph of $K_{P}$ with line set $E_{k}$ for $k=1, \ldots, t$. It remains to be shown that each $H_{k}$ is isomorphic to $H_{1}$. But this is immediate for $1 \leqslant k \leqslant t / 2$, exactly as in the proof of Lemma 1 , part (i), that the $G_{k}$ were 
all isomorphic. Similarly, $H_{i} \cong H_{t}$ whenever $t / 2<i<t$. The principle is illustrated in Figure 9, in which a sample sequence $H_{1}, H_{2}, H_{3}$ is shown for $p=16$ and $t=10$.
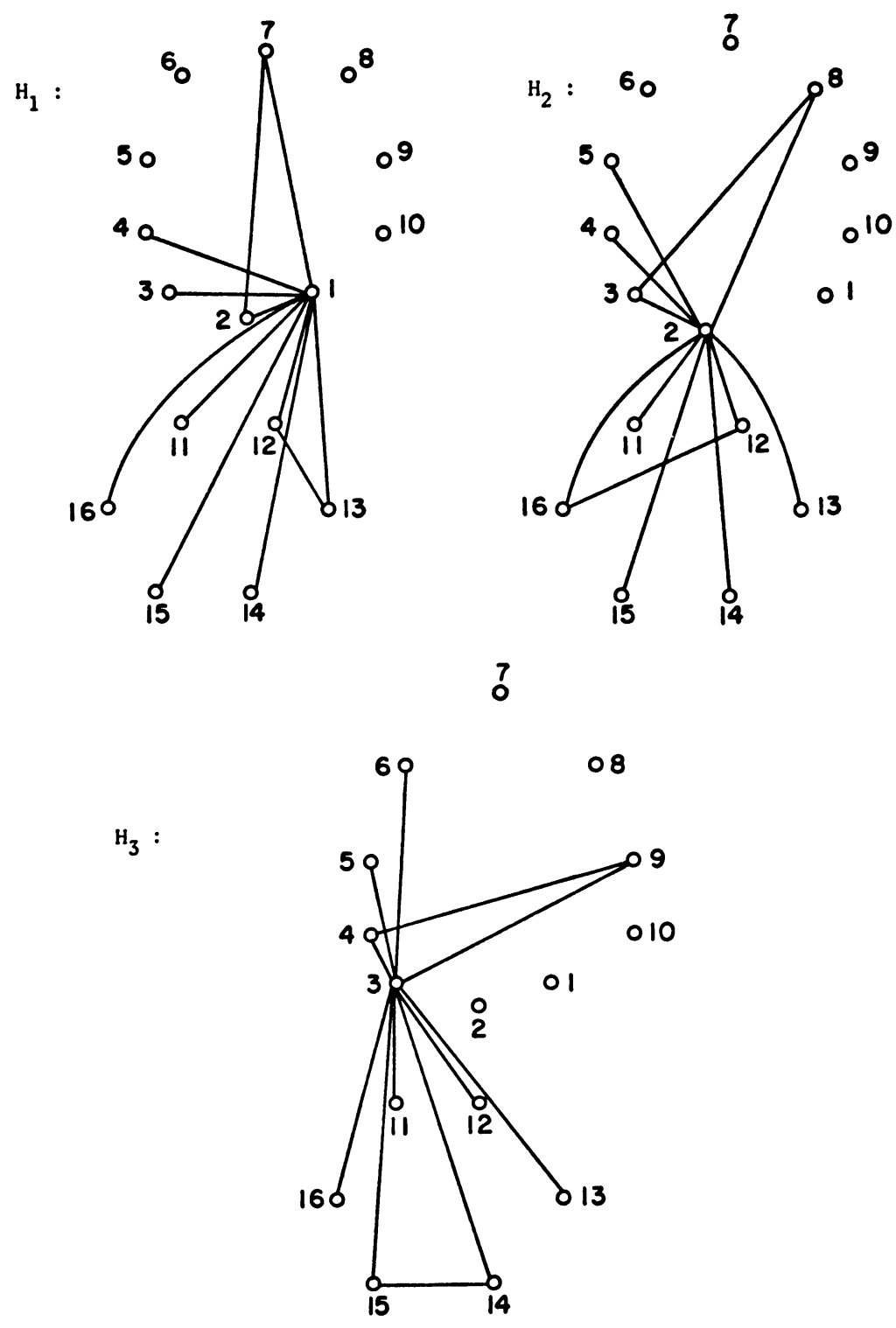

FIGURE 9. Three graphs in an isomorphic factorisation of $K_{16}$

It only remains to show that $H_{t} \cong H_{1}$. Recall that $F_{t}$ is the line set of the graph $G_{t} \cong G \cup K_{2}$ on the points of $K_{r}$. Thus we may choose a line $e_{1}=\left\{x_{1}, y_{1}\right\}$ in $F_{t}$ which is not adjacent to any other line in $F_{t}$. On the other 
hand, let $e_{2}=\left\{x_{2}, y_{2}\right\}=\left(\phi^{\prime}\right)^{t-1}\{i, j\}$. Then from the definition of $E_{k}$ for $k>t / 2, e_{2} \notin E_{t}$. Furthermore, if $E_{t}^{\prime}=\left\{e_{2}\right\} \cup E_{t}-\left\{e_{1}\right\}$, we have that $E_{t}^{\prime}=$ $\left(\phi^{\prime}\right)^{t-1} S \cup\{\{t, x\}: t+1 \leqslant x \leqslant p\} \cup F_{t}^{\prime}$ where $F_{t}^{\prime}=F_{t}-\left\{e_{1}\right\}$ induces the spanning subgraph $G \cup 2 K_{1}$ of $K_{r}$. So if $H_{t}^{\prime}$ is the subgraph of $K_{p}$ with line set $E_{t}^{\prime}$, the argument above showing that $H_{1} \cong H_{k}$ for $2<k<t / 2$ applies here to show that $H_{t}^{\prime} \cong H_{1}$. Finally, since we have chosen $S$ in such a manner that the only lines within $S$ which are adjacent to $\{i, j\}$ are $\{1, i\}$ and $\{1, j\}$, and since no lines in $E_{t}$ are adjacent to $\left\{x_{1}, y_{1}\right\}$ other than $\left\{t, x_{1}\right\}$ and $\left\{t, y_{1}\right\}$, we have an isomorphism between $H_{t}$ and $H_{t}^{\prime}$ induced by interchanging $x_{1}$ with $x_{2}$ and $y_{1}$ with $y_{2}$. This completes the proof of Lemma 3. The latter isomorphism is illustrated for $p=16$ and $t=10$ in Figure 10 with a sample pair $H_{t}, H_{t}^{\prime}$. In this case, $x_{2}=1$ and $y_{2}=6$, and $G$ is $K_{2} \cup 2 K_{1}$. Since $G$ does satisfy the hypotheses of the lemma, it follows that the graph pictured in Figure 10 is an element of $K_{16} / 10$.

5

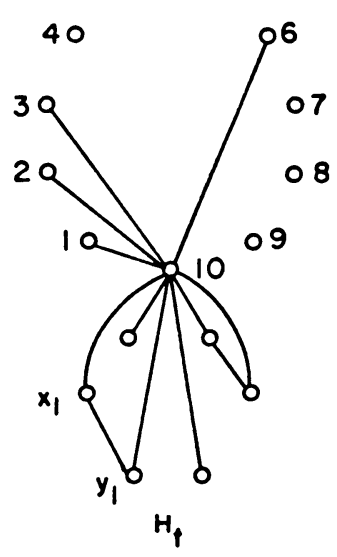

5

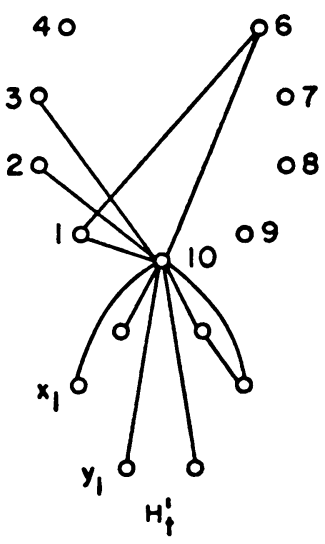

FIGURE 10. Two copies of a graph which divides $K_{16}$

In order to apply Lemma 3 to prove the Divisibility Theorem for $K_{p}$ and $t$ when $t>4, t$ is even, and $p \equiv t+r(\bmod 2 t)$ for $0<r<t$, it is necessary to show the existence of a factorisation of $K_{r}$ satisfying the hypotheses of the lemma. This is done in the next corollary by using the labelling of the lines of $K_{r}$ which was defined in the proof of Lemma 2.

COROLlaRY 2. Suppose we have $t(2 n+1)=m(m-1)$ and $n \leqslant(m-3) / 2$ where $n$ is a nonnegative integer and $m$ and $t$ are positive integers. Then there is a factorisation of $K_{m}$ into $t / 2$ copies of $G \cup 2 K_{1}$ and $t / 2$ copies of $G \cup K_{2}$, where $G$ is the graph $n K_{2}$ plus isolated points. 


\section{Proof.}

Case 1. $n<(m-3) / 2$. Label the lines of $K_{m}$ using the method described in the proof of Lemma 2, which depends on whether $m$ is odd or even. We partition the lines of $K_{m}$ into $t / 2$ sets of $n$ lines and $t / 2$ sets of $n+1$ lines such that the labels of the lines in each set are consecutive. Since $n+1 \leqslant(m$ - 2)/2, Lemma 2 implies that no set of $n+1$ consecutive lines contains an adjacent pair. Thus our partition gives the required factorisation of $K_{m}$.

Case 2. $n=(m-3) / 2$. Then $t(m-2)=m(m-1)$, and thus $(m-$ 2) $\mid m(m-1)$. Since $(m-1)$ is prime to $(m-2)$, this means $(m-2) \mid m$. Thus $m=3$ or 4 . However $m=4$ is impossible because $n=(m-3) / 2$ is an integer. So the only possibility in this case is that $m=3$, and thus $n=0$ and $t=6$. As $K_{2} \cup K_{1} \in K_{3} / 3$, it suffices for the corollary to let $G=K_{1}$. This completes the proof of Corollary 2.

We are finally ready to prove the Divisibility Theorem.

Proof of THEOREM 4. As stated previously, it is immediate that $t$ divides $p(p-1) / 2$ if $K_{p}$ is divisible by $t$. So assume that $t \mid p(p-1)$. Corollary 1 has covered the case of odd $t$, so assume further that $t$ is even.

Let $r^{\prime}$ be the remainder on dividing $p$ by $2 t$, so that $0 \leqslant r^{\prime}<2 t$. Then $t \mid r^{\prime}\left(r^{\prime}-1\right) / 2$. If $r^{\prime}<t$ the argument of Corollary 1 is sufficient. In case $r^{\prime} \geqslant t$, let $r=r^{\prime}-t$. Then for $t$ to divide $p(p-1) / 2, r$ is necessarily in the range $1<r<t$. Furthermore, we may assume $t>4$, since if $t \leqslant 4$ and $p \equiv t+r(\bmod 2 t)$ there is no case in which $t \mid p(p-1) / 2$. Thus to find an element of $K_{r^{\prime}} / t$, Lemma 3 is applicable if a suitable graph $G$, satisfying the requirements of the lemma, can be found.

Since $t \mid r^{\prime}\left(r^{\prime}-1\right) / 2$, we find that $t \mid(r(r-1)-t) / 2$. Let $n$ be the nonnegative integer $r(r-1) / 2 t-\frac{1}{2}$. Then $n<(r-2) / 2$ because $r / t<1$. As $n$ is an integer, $n \leqslant(r-3) / 2$. We can now use Corollary 2 with $m=r$ because $t(2 n+1)=r(r-1)$. This gives the factorisation of $K_{r}$ required for the application of Lemma 3 to show that $K_{r}$ is divisible by $t$. From Lemma 1 it follows in turn that $K_{p}$ is divisible by $t$, which concludes the proof.

5. Related problems. In this concluding section we discuss eight related problem areas, using Roman numerals for subsections. There have been previous studies of isomorphic factorisations in which the factors have certain prescribed properties. One such property is the diameter. In I some results concerning isomorphic factorisations into factors with a given diameter are described. The enumeration problem for isomorphic factors is posed in II. The well-known Kirkman's Schoolgirl Problem is a special case of an isomorphic factorisation in which the factors are required to be regular of degree 2, as discussed in III.

In IV we consider the divisibility of $K_{p}$ by a graph $G$ consisting of a cycle and isolated points. When the cycle is a triangle, we have a Steiner Triple 
System. The problem of deciding which stars divide $K_{p}$ has been settled (V), but the more general problem for trees (VI) is still open. Subsection VII discusses progress toward generating the graphs in $K_{p} / t$. We conclude VIII with a brief preview of forthcoming papers in this series.

I. Factors of given diameter. Ringel [11] showed that every nontrivial self-complementary graph has diameter 2 or 3. Kotzig and Rosa [8] studied more general isomorphic factorisations of $K_{p}$ into subgraphs with given diameter. In particular they investigated $K_{p} / 3$ so that $p=3 n$ or $3 n+1$. For $n>2$, they found that $K_{p} / 3$ contains graphs of diameters $3,4, \ldots, n+2$. For diameter 2, they noted that $K_{p} / 3$ cannot contain a graph of diameter 2 when $p<12$ and does contain such a graph for $p>13$. It is probable that there is no graph of diameter 2 in $K_{12} / 3$. This question might remain open until a clever computer program is devised to settle it.

II. Enumeration of isomorphic factors. Read [10] derived a formula for the number of self-complementary $p$-point graphs, that is, the number of graphs in $K_{p} / 2$. A corresponding formula for the cardinality of $K_{p} / t$ when $t \geqslant 3$ appears to be utterly intractable.

III. Factors regular of degree 2. Hell, Kotzig and Rosa [5] studied isomorphic factorisations of $K_{p}$ where each factor is regular of degree 2. Of course such a factor is the union of point-disjoint cycles. They consider in particular the situation when all the cycles have the same length $s$. One of their results is that if $K_{p}$ has a factor of this form then $s \mid p$, and $s$ and $p / s$ are both odd, and they conjecture that the converse holds. The special case $s=3$ is well known as Kirkman's Schoolgirl Poblem. It was finally settled by Ray-Chaudhuri and Wilson [9].

IV. Steiner triple systems. When a graph $G$ which divides $K_{p}$ consists of one triangle and $p-3$ isolated points, $G=K_{3} \cup(p-3) K_{1}$, the isomorphic factorisation is called a Steiner Triple System (STS) of order p; see [14, pp. 99-101]. It is easy to see that every STS has order $p \equiv 1$ or $3(\bmod 6)$ and STS's of every such order have been constructed. It has been shown that the number of STS's of order 3, 7, 9, 13, 15 is 1, 1, 1, 2, 80 respectively, but for $p \geqslant 19$ this enumeration problem is also intractable; compare Problem II above.

The corresponding problem when a triangle $C_{3}$ is replaced by a pentagon $C_{5}$ is studied by Rosa and Huang [13]. They find that $C_{5}$ divides $K_{p}$ if and only if $p \equiv 1$ or $5(\bmod 10)$, that is, $5 \mid p(p-1) / 2$.

V. Stars. Isomorphic factorisations of $K_{p}$ in which each factor is a star $K_{1, n}$ (together with $p-n-1$ isolates to have a spanning subgraph) were investigated independently by Hogarth [6] and Yamamoto et al. [18]. In [6], only the cases $n=6$ and 10 were considered while in [18] it was shown that such an isomorphic factorisation exists if and only if $n \mid p(p-1) / 2$ and $p \geqslant 2 n$. 
VI. Trees. More general than the preceding theorem for stars was the problem posed by Ringel [12] when he conjectured that every tree $T$ with $q$ lines (plus $q$ isolated points for dimensional correctness) divides $K(2 q+1)$. Kotzig [7] found a related result to the effect that almost all trees satisfy this conjecture.

VII. One-third of $K_{p}$. When does the method of Theorem 1 give all the graphs in $K_{p} / t$ ? It always does for $t=2$ as shown by Ringel [11] and Sachs [15] in their works on the structure of self-complementary graphs.

In Theorems 2 and 3 , we found the entire sets $K_{6} / 3$ and $K_{7} / 3$ by the method. We guess that this approach will also yield all the graphs in $K_{9} / 3$ but probably not all of $K_{10} / 3$. Thus we ask for the minimum $p$ such that $K_{p} / 3$ is not generated by this method.

VIII. Isomorphic factorisations of graphs other than $K_{p}$. In future papers in this series, we plan to investigate isomorphic factorisations of complete multipartite graphs and trees. We also propose to study the automorphism group of an isomorphic factorisation as a permutation group which acts on the factors.

In [13] it was pointed out that the construction of isomorphic factorisations of complete multigraphs is tantamount to finding certain block designs. We plan to collaborate with W. D. Wallis on a systematic investigation of this correspondence.

There is already a body of literature concerning isomorphic factorisations of complete graphs into a given graph, as seen from the viewpoint of design theory. A recent review of publications in this area is contained in [1]. In the same volume it is shown by Wilson [17] that for any arbitrary graph $G$ with $q$ lines, $G \mid K_{p}$ for all sufficiently large $p$ (depending on $G$ ) provided that $q \mid p(p-1) / 2$ and $p-1$ is a multiple of the greatest common divisor of the degrees of the points of $G$. Thus these obvious necessary conditions for an isomorphic factorization of $G$ into $K_{p}$ are also sufficient when $p$ is big enough.

ACKNOWLEDGEMENT. The authors thank the referee for several helpful suggestions.

\section{REFERENCES}

1. J. C. Bermond and D. Sotteau, Graph decompositions and G-designs, Proceedings of the Fifth British Combinatorial Conference, (C. St. J. A. Nash-Williams and J. Sheehan, eds.), Utilities Math., Winnipeg, 1976, pp. 53-72.

2. L. Guidotti, Sulla divisibilità dei grafi completi, Riv. Mat. Univ. Parma 1 (1972), 231-237.

3. F. Harary, Graph theory, Addison-Wesley, Reading, Mass., 1969.

4. F. Harary and E. M. Palmer, Graphical enumeration, Academic Press, New York, 1973.

5. P. Hell, A. Kotzig and A. Rosa, Some results on the Oberwolfach problem: Decomposition of complete graphs into isomorphic quadratic factors, Aequationes Math. 12 (1975), 1-5.

6. P. C. Hogarth, Decomposition of complete graphs into 6-stars and into 10-stars, 
Combinatorial Mathematics III, Springer-Verlag, Berlin, 1975, pp. 136-142.

7. A. Kotzig, On certain vertex valuations of finite graphs, Utilitas Math. 4 (1973), 261-290.

8. A. Kotzig and A. Rosa, Decomposition of complete graphs into isomorphic subgraphs with a given diameter, Bull. London Math. Soc. 7 (1975), 51-57.

9. D. K. Ray-Chaudhuri and R. M. Wilson, Solution of Kirkman's schoolgirl problem, Proc. Sympos. Pure Math., vol. 19, Combinatorics, Amer. Math. Soc., Providence, R. I., 1971, pp. 187-203.

10. R. C. Read, On the number of self-complementary graphs and digraphs, J. London Math. Soc. 38 (1963), 99-104.

11. G. Ringel, Selbstkomplementäre Graphen, Arch. Math. 14 (1963), 354-358.

12. _ Problem 25, Theory of Graphs and Its Applications, (M. Fiedler, ed.), Academic Press, New York, 1964, p. 162.

13. A. Rosa and C. Huang, Another class of balanced block designs: balanced circuit designs, Discrete Math. 12 (1975), 269-293.

14. H. J. Ryser, Combinatorial mathematics, Carus Monograph 14, Math. Assoc. Amer., 1963.

15. H. Sachs, Uber selbstkomplementäre Graphen, Publ. Math. Debrecen 9 (1962), 270-288.

16. G. J. Simmons and J. A. Davis, Pair designs, Comm. Statist. 4 (1975), 255-272.

17. R. M. Wilson, Decomposition of complete graphs into subgraphs isomorphic to a given graph, Proceedings of the Fifth British Combinatorial Conference, (C. St. J. A. Nash-Williams and J. Sheehan, eds.) Utilitas Math., Winnipeg, 1976, pp. 647-659.

18. S. Yamamoto, H. Ikeda, S. Shige-eda, K. Ushio and N. Hamada, On claw-decomposition of complete graphs and complete bigraphs, Hiroshima Math. J. 5 (1975), 33-42.

Department of Mathematics, University of Michigan, AnN Arbor, Michigan 48109 (Current address of Frank Harary)

Department of Mathematics, University of Newcastle, New South Wales, 2308, Australin (Current address of R. W. Robinson and N. C. Wormald) 